October 1998

UCB-PTH-98/48

LBNL-42383

\title{
Dualities of the Matrix Model from T-Duality of the Type II Stringf
}

\author{
Daniel Brace'], Bogdan Morariuf and Bruno Zumino \\ Department of Physics \\ University of California \\ and \\ Theoretical Physics Group \\ Lawrence Berkeley National Laboratory \\ University of California \\ Berkeley, California 94720
}

\begin{abstract}
We investigate in the Matrix theory framework, the subgroup of dualities of the DLCQ of M-theory compactified on three-tori, which corresponds to T-duality in the auxiliary Type II string theory. We show how these dualities are realized in the supersymmetric YangMills gauge theories on dual noncommutative three-tori.
\end{abstract}

PACS 11.15.-q, 11.25.-w.

${ }^{*}$ This work was supported in part by the Director, Office of Energy Research, Office of High Energy and Nuclear Physics, Division of High Energy Physics of the U.S. Department of Energy under Contract DE-AC03-76SF00098 and in part by the National Science Foundation under grant PHY-95-14797

$\dagger$ email address: brace@thwk2.lbl.gov

${ }_{\ddagger}^{\ddagger}$ email address: morariu@thsrv.lbl.gov

$\S$ email address: zumino@thsrv.lbl.gov 


\section{Disclaimer}

This document was prepared as an account of work sponsored by the United States Government. While this document is believed to contain correct information, neither the United States Government nor any agency thereof, nor The Regents of the University of California, nor any of their employees, makes any warranty, express or implied, or assumes any legal liability or responsibility for the accuracy, completeness, or usefulness of any information, apparatus, product, or process disclosed, or represents that its use would not infringe privately owned rights. Reference herein to any specific commercial products process, or service by its trade name, trademark, manufacturer, or otherwise, does not necessarily constitute or imply its endorsement, recommendation, or favoring by the United States Government or any agency thereof, or The Regents of the University of California. The views and opinions of authors expressed herein do not necessarily state or reflect those of the United States Government or any agency thereof, or The Regents of the University of California.

Lawrence Berkeley National Laboratory is an equal opportunity employer. 


\section{Introduction}

It was conjectured by Susskind [1] that each momentum sector of the discrete light cone quantization (DLCQ) of M-theory is described by a maximally supersymmetric Matrix model [2, 3, 4] with the momentum identified with the rank of the gauge group. The conjecture was further clarified by Sen and Seiberg [5, 6]. They used an infinite boost and a compensating rescaling to show that the DLCQ Hamiltonian of the original M-theory is given by the Hamiltonian of an auxiliary M-theory compactified on a vanishingly small

space-like circle of radius $R$. This is then equivalent to a weakly coupled Type IIA string theory, which will be referred to, following Sen [7], as the auxiliary Type II string theory. At the same time, the original light-cone momentum is mapped into Ramond-Ramond D0 brane charge. The string coupling and string mass scale are given by the $R \rightarrow 0$ limit of

$$
g_{S}=M_{P}^{3 / 2}(L R)^{3 / 4}, m_{S}=M_{P}^{3 / 2} L^{3 / 4} R^{-1 / 4} .
$$

In this limit as proposed by Witten [8], and discussed extensively in [9], the dynamics of $n$ D0 branes is determined by the maximally supersymmetric Matrix model [2, 3, 团.

Toroidal compactification of M-theory can be obtained by considering Matrix theory on the covering space of the torus and imposing a periodicity constraint on the dynamical variables [10, 11, 12]. The constrained system is formally equivalent to a $U(n)$ super Yang-Mills (SYM) gauge theory on a dual torus. On the other hand upon compactification on a $d$-dimensional torus $T^{d}$ M-theory has additional moduli from the three form of eleven dimensional supergravity. Connes, Douglas and Schwarz [13], conjectured that these moduli correspond to the deformation parameters $\Theta_{i j}$ of a noncommutative super Yang-Mills (NCSYM) gauge theory. Further studies of this subject followed in 114, 15, 16, 17, 18, 19, 20, 21, 22, 23, 24, 25, 26, 27.

In [13], where compactification on a two-torus was considered in some detail, it was suggested that the $S L(2, \mathbf{Z})$ noncommutative duality group of 
the NCSYM gauge theory [28, 29, 30, 31, 32] corresponds to the T-duality in the DLCQ direction and one of the space-like compact directions of Mtheory. Later Rieffel and Schwarz [20] showed that NCSYM gauge theories on higher dimensional tori have an $S O(d, d \mid \mathbf{Z})$ duality, and conjectured that this is the realization, in the NCSYM theory, of the auxiliary Type II string theory T-duality.

In this paper we investigate this conjecture for compactifications on a three-torus. First we extend the method used in [22, 26] to construct twisted bundles to the three-torus. Then we show explicitly how to construct an action of the duality group $S O(3,3 \mid \mathbf{Z})$ on NCSYM theories. Under these duality transformations the rank of the gauge group and the magnetic flux numbers transform together in a Weyl spinor representation, and the deformation parameters transform by fractional transformations. We also obtain the transformation properties, under the duality group, of the gauge coupling and the metric. We can then directly compare these relations with the string theory T-duality predictions.

In the next section we review the standard toroidal Matrix compactification leading to a SYM gauge theory on the dual torus. Then we present the conjecture [13], that in the presence of nonvanishing NS antisymmetric moduli $B_{i j}$, the translation generators implementing the quotient condition do not commute, such that Matrix compactification leads to a noncommutative super Yang-Mills gauge theory on a dual noncommutative torus.

In Section 3, we study adjoint quantum bundles on noncommutative tori of arbitrary dimension which admit a constant curvature which is not valued in the $s u(n)$ subalgebra and have transition functions of a special simple form.

In Section 1 we show how to expand the sections of the adjoint bundle of a $U(n)$ gauge theory in terms of matrix valued functions on a dual noncommutative torus. The dual deformation parameter $\Theta^{\prime}$ lies on the same $S O(d, d \mid \mathbf{Z})$ orbit as the original $\Theta$. We perform most of the calculations on tori of arbitrary dimension, but later in the paper we concentrate on the two 
and three-tori.

In Section 5, we describe the quantum bundles corresponding to the two dimensional compactification. We rewrite some of the known two dimensional relations in a form that admits immediate generalization to higher dimensions. Section 6 contains the solution for arbitrary bundles over three dimensional tori.

In Section 7, we consider the noncommutative SYM action on a twisted quantum bundle after a brief description of the quantum integral.

In Section 8, we show how to manipulate the NCSYM action of a $U(n)$ theory on a twisted three-torus such that it is formally equivalent to a $U(q)$ action on a trivial bundle over a dual torus, where $q$ is the greatest common divisor of $n$ and the magnetic fluxes of the bundle. More generally, two NCSYM theories are equivalent if their rank and magnetic fluxes and deformation parameters are on the same orbit of $S O(3,3 \mid \mathbf{Z})$. The rank and the magnetic fluxes transform in an integral Weyl spinor representation of the group. Related results were obtained in [24] using a more abstract mathematical language. In the last section we show that this duality is the low energy remnant of the T-duality of Type II string theory.

Finally in the appendix we prove a theorem showing that the chiral spinor representations of $S O(d, d \mid \mathbf{Z})$ are integral, and also show that the spinor representation of $S O(3,3 \mid \mathbf{Z})$ is in fact $S L(4, \mathbf{Z})$.

\section{Matrix Compactification}

In this section we present a review of Matrix theory compactification. In the limit of large string mass the dynamics of $n$ D0 branes, in uncompactified space-time, is determined by the maximally supersymmetric Matrix action [2, 3, 4,

$\mathcal{S}^{D 0}=\frac{1}{2 g_{S}} \int d t \operatorname{tr}\left(\sum_{M} \dot{X}^{M} \dot{X}^{M}+\frac{1}{(2 \pi)^{2}} \sum_{M<N}\left[X^{M}, X^{N}\right]\left[X^{M}, X^{N}\right]+\right.$ fermions $)$. 
This action is obtained by dimensional reduction of the ten dimensional $\mathcal{N}=1$ SYM gauge theory. Alternatively we could work with the IKKT functional [33] obtained by dimensionally reducing, in all directions including time, the Euclidean ten dimensional SYM action.

The compactification of Matrix theory on a $d$-dimensional torus is obtained by considering an infinite number of $\mathrm{D} 0$ branes living on $\mathbf{R}^{d}$, the covering space of the torus, and then imposing the following quotient conditions [10, 11].

$$
\begin{gathered}
\mathcal{U}_{i}^{-1} X^{I} \mathcal{U}_{i}=2 \pi e_{i}^{I}+X^{I}, i, I=1, \ldots, d, \\
\mathcal{U}_{i}^{-1} X^{a} \mathcal{U}_{i}=X^{a}, a=d+1, \ldots, 9 .
\end{gathered}
$$

Here $I$ runs over the compact directions, and the $e_{i}^{I}$ form a basis defining the compactification lattice. The $\mathcal{U}_{i}$ 's are unitary operators. One can define new matrix coordinates

$$
X^{i}=e_{I}^{i} X^{I},
$$

which obey the simpler quotient conditions

$$
\mathcal{U}_{i}^{-1} X^{j} \mathcal{U}_{i}=2 \pi \delta_{i}^{j}+X^{j} .
$$

In terms of the new variables the action takes the form

$$
\begin{gathered}
\mathcal{S}^{D 0}=\frac{1}{2 g_{S}} \int d t \operatorname{Tr}\left(G_{i j} \dot{X}^{i} \dot{X}^{j}+\frac{1}{2} \frac{1}{(2 \pi)^{2}} G_{i j} G_{k l}\left[X^{i}, X^{k}\right]\left[X^{j}, X^{l}\right]+\right. \\
\sum_{a} \dot{X}^{a} \dot{X}^{a}+\frac{1}{(2 \pi)^{2}} \sum_{a} G_{i j}\left[X^{i}, X^{a}\right]\left[X^{j}, X^{a}\right]+ \\
\left.\frac{1}{(2 \pi)^{2}} \sum_{a<b}\left[X^{a}, X^{b}\right]\left[X^{a}, X^{b}\right]+\text { fermions }\right),
\end{gathered}
$$

where we have introduced the metric $G_{i j}=\sum_{I} e_{i}^{I} e_{j}^{I}$. In (2), the trace over infinite dimensional matrices is formally divided by the infinite order the quotient group $\mathbf{Z}^{d}$. 
The original solution of the quotient condition assumed that the translation operators commute

$$
\left[\mathcal{U}_{i}, \mathcal{U}_{j}\right]=0 .
$$

The standard way to solve (11) is to introduce an auxiliary Hilbert space on which $X^{i}$ 's and $\mathcal{U}_{i}$ 's act. In the simplest case this is taken to be the space of functions on a $d$-dimensional torus taking values in $\mathbf{C}^{n}$. Then if we let the $\mathcal{U}_{i}$ 's be the generators of the algebra of functions on the torus

$$
\mathcal{U}_{i}=e^{i \sigma_{i}},
$$

where $\sigma_{i}$ are coordinates on the covering space of the torus, the $X^{i}$ 's satisfying (1) must be covariant derivatives

$$
X^{j}=-2 \pi i D^{j}=-2 \pi i\left(\partial^{j}-i A^{j}\left(\mathcal{U}_{k}\right)\right) .
$$

The partial derivative is with respect to $\sigma_{j}$, and $A^{j}$ are $n$-dimensional hermitian matrices. The action (21) can be rewritten as a $d$-dimensional SYM action, by replacing the $X^{i}$ 's with covariant derivatives as above, and rewriting the trace over the infinite dimensional matrices as

$$
\operatorname{Tr}=\int \frac{d^{d} \sigma}{(2 \pi)^{d}} \operatorname{tr} .
$$

Here $\operatorname{tr}$ is an $n$-dimensional trace, and the new coordinates $\sigma_{i}$ are to be integrated from zero to $2 \pi$. The action becomes

$$
\begin{aligned}
\mathcal{S}^{D 0}= & \frac{(2 \pi)^{2-d}}{4 g_{S} \sqrt{\operatorname{det}\left(G^{i j}\right)}} \int d t \int d^{d} \sigma \sqrt{\operatorname{det}\left(G^{i j}\right)} \operatorname{tr}\left(G_{\mu \nu} G_{\xi \rho}\left[D^{\mu}, D^{\xi}\right]\left[D^{\nu}, D^{\rho}\right]-\right. \\
& \left.\sum_{a} G_{\mu \nu}\left[D^{\mu}, X^{a}\right]\left[D^{\nu}, X^{a}\right]+\sum_{a<b}\left[X^{a}, X^{b}\right]\left[X^{a}, X^{b}\right]+\text { fermions }\right),
\end{aligned}
$$

where the scalar fields $X^{a}$ have been rescaled by a factor of $2 \pi$. We have 
written the action in standard form ${ }^{\text {s }}$ so that we can read off the SYM gauge coupling

$$
g_{S Y M}^{2}=g_{S}(2 \pi)^{d-2} \sqrt{\operatorname{det}\left(G^{i j}\right)} .
$$

Thus the gauge coupling $g_{S Y M}^{2}$ equals the string coupling on the T-dual torus. The square root factor accounts for the expected dilaton shift under T-duality.

Following [13] we consider the general case when the unitary operators $\mathcal{U}_{i}$ do not commute. Consistency of the quotient conditions requires that the $\mathcal{U}_{i}$ 's must commute up to a phase

$$
\mathcal{U}_{i} \mathcal{U}_{j}=e^{-2 \pi i \Theta_{i j}} \mathcal{U}_{j} \mathcal{U}_{i}
$$

Connes, Douglas and Schwarz conjectured that the deformation parameters $\Theta$ correspond to certain moduli of the compactification of the DLCQ of M theory on tori. If $\gamma^{i j-}$ represents a three cycle wrapped around the transversal directions $x^{i}$ and $x^{j}$ and the light cone direction $x^{-}$, then

$$
\Theta_{i j}=\frac{1}{(2 \pi)^{3}} \int_{\gamma^{i j-}} C,
$$

where $C$ is the antisymmetric three form of eleven dimensional supergravity. Written in terms of the auxiliary type IIA string theory variables,

$$
\Theta_{i j}=\frac{1}{(2 \pi)^{2}} \int_{\gamma^{i j}} B
$$

where $B$ is the NS two form. In the noncommutative case it is convenient to introduce another set of translation operators $U_{i}$ which satisfy

$$
U_{i} U_{j}=e^{2 \pi i \Theta_{i j}} U_{j} U_{i}
$$

\footnotetext{
${ }^{a}$ Note that the positions of all the indices are switched. For example the metric has upper indices. This just reflects that we have performed a T-duality under which the metric is replaced with the inverse metric. Another way to understand the index position is that T-duality is a canonical transformation which exchanges coordinates and momenta and therefore reverses the index structure.
} 
The $U_{i}$ 's generate the algebra of functions on a quantum torus. We will denote this algebra, $\mathcal{A}_{\Theta}$. For an expanded discussion of this and other issues in noncommutative geometry see [34, 35]. This algebra can be realized as a subalgebra of the quantum plane algebra, which is generated by $\sigma_{i}$ satisfying

$$
\left[\sigma_{i}, \sigma_{j}\right]=-2 \pi i \Theta_{i j}
$$

Then we realize the generators of $\mathcal{A}_{\Theta}$ as

$$
U_{i} \stackrel{\text { def }}{=} e^{i \sigma_{i}} .
$$

To realize the $\mathcal{U}_{i}$ generators we also introduce partial derivatives satisfying $\left.\right|^{\circ}$

$$
\left[\partial^{i}, \sigma_{j}\right]=\delta_{j}^{i},\left[\partial^{i}, \partial^{j}\right]=0 .
$$

Now we can write the $\mathcal{U}_{i}$ generators as

$$
\mathcal{U}_{i}=e^{i \sigma_{i}-2 \pi \Theta_{i j} \partial^{j}}
$$

Note that both $\sigma_{i}$ and $\partial^{i}$ act as translation generators on the $\sigma_{i}$ 's, and the exponent in the $\mathcal{U}_{i}$ 's is just the linear combination that commutes with all the $\sigma_{i}$ 's. Thus

$$
\left[\mathcal{U}_{i}, U_{j}\right]=0 .
$$

For vanishing $\Theta$ we see that $U_{i}$ and $\mathcal{U}_{i}$ coincide.

The simplest example of solutions of the quotient conditions (11) are quantum connections on trivial bundles

$$
X^{j}=-2 \pi i D^{j}=-2 \pi i\left(\partial^{j}-i A^{j}\left(U_{k}\right)\right) .
$$

In the noncommutative case the matrix elements of $A^{j}$ are elements of $\mathcal{A}_{\Theta}$. Again using the representation (7) of $X^{i}$ in the Matrix model action we obtain a NCSYM action [36]. However we will postpone writing this action until we study more general solutions which are connections on nontrivial bundles.

\footnotetext{
${ }^{b}$ Just as in the classical case, one can also introduce quantum exterior forms $d \sigma_{i}$, which anti-commute with each other and commute with all other variables.
} 


\section{Twisted Quantum Bundles on Tori}

In this section we construct quantum $U(n)$ bundles on $d$-dimensional noncommutative tori which admit constant curvature connections with vanishing $s u(n)$ curvature. This is done by finding explicit transition functions compatible with such a connection. We employ a method which is a straightforward generalization of [22, 26]. Using a gauge transformation the constant curvature connection can be brought into the form

$$
\nabla^{i}=\partial^{i}+i F^{i j} \sigma_{j}
$$

where $F$ is an antisymmetric matrix. We define the constant curvature to be

$$
\mathcal{F}_{(0)}^{j k}=i\left[\nabla^{j}, \nabla^{k}\right]
$$

and using the commutation relations (6) one can calculate

$$
\mathcal{F}_{(0)}=(2 F+2 \pi F \Theta F) \text {. }
$$

In general, such a connection can only exist on a non-trivial bundle. One can introduce transition functions $\Omega_{i}$ such that the connection satisfies the twisted boundary conditions

$$
\nabla^{i}\left(\sigma_{m}+2 \pi \delta_{m}^{j}\right)=\Omega_{j}\left(\sigma_{m}\right) \nabla^{i}\left(\sigma_{m}\right) \Omega_{j}^{-1}\left(\sigma_{m}\right)
$$

We can try to find solutions for the transition functions of the form

$$
\Omega_{j}=e^{i P^{j l} \sigma_{l}} W_{j}
$$

where $P$ is an arbitrary constant $d$-dimensional matrix and the $W_{i}$ 's are constant, unitary $n$-dimensional matrices. The boundary conditions (9) imply the following equivalent relations

$$
\begin{gathered}
P=(1+2 \pi F \Theta)^{-1} 2 \pi F=2 \pi F(1+\Theta 2 \pi F)^{-1}, \\
2 \pi F=P(1-\Theta P)^{-1}=(1-P \Theta)^{-1} P .
\end{gathered}
$$


Note that $P$ must be antisymmetric because of our gauge choice. Consistency of the transition functions of the bundle requires

$$
\Omega_{j}\left(\sigma_{m}+2 \pi \delta_{m}^{i}\right) \Omega_{i}\left(\sigma_{m}\right)=\Omega_{i}\left(\sigma_{m}+2 \pi \delta_{m}^{j}\right) \Omega_{j}\left(\sigma_{m}\right)
$$

which is known in the mathematical literature as the cocycle condition. In our case the cocycle condition implies

$$
W_{i} W_{j}=e^{-2 \pi i M^{i j} / n} W_{j} W_{i},
$$

where the antisymmetric matrix $M$ is given by

$$
M=n(2 P-P \Theta P) .
$$

By taking the determinant of both sides of (11) one finds that $M$ must have integer entries. In the classical case $M^{i j}$ corresponds to the value of the first Chern class on the $(i j)$ two-cycle of the torus. In the auxiliary Type IIA string theory, $M$ is interpreted as D2 brane winding. This interpretation remains true in the quantum case.

Let $q$ be the greatest common divisor of $n$ and the nonvanishing entries of $M$

$$
q=\operatorname{gcd}\left(n, M^{i j}\right) .
$$

Next we define $\tilde{n}$ and $\widetilde{M}$ which have relatively prime entries

$$
n=q \tilde{n}, \quad M=q \widetilde{M} .
$$

It is convenient to consider $W_{i}$ 's which have block diagonal form with $q$ identical blocks along the diagonal

$$
W_{i}=\left(\begin{array}{ccc}
\widetilde{W}_{i} & & \\
& \ddots & \\
& & \widetilde{W}_{i}
\end{array}\right) .
$$

Here $\widetilde{W}_{i}$ are $\tilde{n}$-dimensional matrices. Alternatively we can write this in tensor product notation

$$
W_{i}=I_{q} \otimes \widetilde{W}_{i}
$$


The transition functions are also block diagonal and can be written

$$
\Omega_{i}=I_{q} \otimes \omega_{i} .
$$

To find explicit boundary conditions, following 't Hooft [37], we make the ansatz

$$
\widetilde{W}_{i}=U^{a^{i}} V^{b^{i}},
$$

where $a^{i}$ and $b^{i}$ are integers and $U$ and $V$ are the clock and shift matrices [38, 37]

$$
U_{k l}=e^{2 \pi i(k-1) / \tilde{n}} \delta_{k, l}, \quad V_{k l}=\delta_{k+1, l}, \quad k, l=1, \ldots, \tilde{n},
$$

and the subscripts are identified with period $\tilde{n}$. They satisfy

$$
U V=e^{-2 \pi i / \tilde{n}} V U \text {. }
$$

Then (11) leads to the following relation

$$
\widetilde{M}^{i j}=\left(a^{i} b^{j}-b^{i} a^{j}\right) \bmod (\tilde{n}) .
$$

For two or three dimensional tori, one can find integers $a^{i}$ and $b^{i}$ such that (14) holds for arbitrary $M$, as will be shown in Sections 5 and 6 . In higher dimensional cases the ansatz is not sufficiently general to describe arbitrary bundles. In particular, we can always perform a change of lattice basis such that the only nonvanishing components of $M$ are $M^{d-1, d}=-M^{d, d-1}$, while in general, an arbitrary antisymmetric matrix can not be brought into such a form. Furthermore, for $d>3$, even in the commutative case, generic bundles do not admit connections with vanishing $s u(n)$ constant curvature. A more general construction could be obtained by allowing for an arbitrary constant curvature connection.

\section{Adjoint Sections on Twisted Bundles}

In this section we analyze the structure of adjoint sections on twisted bundles. The scalar and fermion fields are examples of such sections. We will also 
write the connection as a sum of a constant curvature connection $\nabla^{i}$, and a fluctuating part $A^{i}$

$$
D^{i}=\nabla^{i}-i A^{i}
$$

Note that $A^{i}$ is also an adjoint section. Since it is the difference between two connections it transforms covariantly under gauge transformations. It should not be confused with a gauge potential. Adjoint sections are $n$-dimensional matrices with entries which are elements of the quantum plane algebra (6) and obey the twisted boundary conditions

$$
\Psi\left(\sigma_{i}+2 \pi \delta_{i}^{j}\right)=\Omega_{j} \Psi\left(\sigma_{i}\right) \Omega_{j}^{-1}
$$

Next we will try to find the general solution of (15) and write it in unconstrained form, reflecting the global properties of the bundle. First consider the simpler example of a $U(n)$ NCSYM on a trivial bundle over a two-torus. Since $\Omega_{i}=1$ we have

$$
\Psi=\sum_{a, b=1}^{n} E^{a b} \otimes\left[\sum_{i_{1} i_{2} \in \mathbf{Z}} \Psi_{i_{1} i_{2}}^{a b} U_{1}^{i_{1}} U_{2}^{i_{2}}\right],
$$

where $E^{a b}$ are $n$-dimensional matrices with one nonzero entry, $\left(E^{a b}\right)_{i j}=\delta_{i}^{a} \delta_{j}^{b}$, and $\Psi_{i_{1} i_{2}}^{a b}$ are $c$-numbers. In other words, each matrix element of the adjoint section is an arbitrary function on the quantum torus. If we consider a twisted $U(n)$ bundle with magnetic flux $m$, such that $n$ and $m$ are relatively prime, one can show [13, 22, 26] that the adjoint sections have the expansion

$$
\Psi=\sum_{i_{1} i_{2} \in \mathbf{Z}} \Psi_{i_{1} i_{2}} Z_{1}^{i_{1}} Z_{2}^{i_{2}}
$$

where now the coefficients $\Psi_{i_{1} i_{2}}$ are $c$-numbers, and $Z_{i}$ are $n$-dimensional matrices with noncommutative entries satisfying

$$
Z_{1} Z_{2}=e^{2 \pi i \theta^{\prime}} Z_{2} Z_{1}
$$

Thus the $Z_{i}$ 's satisfy the commutation relations of a generators of the quantum torus. This shows that the set of sections is isomorphic to the set of 
functions on a dual torus, and is very similar to the set of adjoint sections of a $U(1)$ NCSYM theory. For two and three dimensional adjoint bundles with arbitrary magnetic fluxes, we will show that the general solution takes the form

$$
\Psi=\sum_{a b=1}^{q} E^{a b} \otimes\left[\sum_{i_{1} i_{2} \ldots i_{d} \in \mathbf{Z}} \Psi_{i_{1} i_{2} \ldots i_{d}}^{a b} Z_{1}^{i_{1}} Z_{2}^{i_{2}} \ldots Z_{d}^{i_{d}}\right], \quad d=2,3 .
$$

Here $E^{a b}$ are $q$ dimensional.

We begin by writing $\Psi$ in tensor notation

$$
\Psi\left(\sigma_{i}\right)=\sum_{a, b=1}^{q} E^{a b} \otimes \Psi^{a b}\left(\sigma_{i}\right),
$$

where $\Psi^{a b}\left(\sigma_{i}\right)$ are $\tilde{n}$-dimensional matrices with noncommutative entries. Imposing the boundary conditions (15) and using (12) we obtain

$$
\Psi^{a b}\left(\sigma_{i}+2 \pi \delta_{i}^{j}\right)=\omega_{j} \Psi^{a b}\left(\sigma_{i}\right) \omega_{j}^{-1}
$$

A less restrictive but very convenient constraint is obtained by shifting $\sigma_{i}$ by $2 \pi \tilde{n}$ using (17)

$$
\Psi^{a b}\left(\sigma_{i}+2 \pi \tilde{n} \delta_{i}^{j}\right)=\omega_{j}^{\tilde{n}} \Psi^{a b}\left(\sigma_{i}\right) \omega_{j}^{-\tilde{n}} .
$$

In (18) all the matrix factors disappear since $U^{\tilde{n}}=V^{\tilde{n}}=1$. The $\sigma_{i}$ dependent exponential of (10) survives and acts like a translation operator due to the commutation relations (6). This implies the following periodicity relation

$$
\Psi^{a b}\left(\sigma_{i}+2 \pi \tilde{n}\left(Q^{-1}\right)_{i}^{j}\right)=\Psi^{a b}\left(\sigma_{i}\right)
$$

where

$$
Q^{-1}=1-P \Theta
$$

Next we try to find solutions of the form

$$
Z_{i}=e^{i \sigma_{j} Q_{k}^{j} N_{i}^{k} / \tilde{n}} U^{s_{i}} V^{t_{i}}, \quad i=1 \ldots d .
$$


Here $s_{j}$ and $t_{j}$ are integers and the exponent was chosen so that it is compatible with the constraint (19) if the matrix $N$ has integer entries. One can show that $Z_{i}$ is compatible with the boundary conditions (17) if

$$
N_{j}^{i}=\left(b^{i} s_{j}-a^{i} t_{j}\right) \bmod (\tilde{n}),
$$

where $a^{i}$ and $b^{i}$ are defined by (14). In the next two sections we will consider in detail the two and three dimensional cases, and find $a^{i}, b^{i}, s_{j}$ and $t_{j}$ such that (14) and (21) hold. Furthermore, for properly chosen integers $a^{i}, b^{i}, s_{j}$ and $t_{j}$, one can show that an arbitrary adjoint section can be expanded in terms of the $Z_{i}$ 's as in (16). For a proof of this statement in two dimensions see [26. It is convenient to define another matrix which will be used shortly,

$$
L_{i j}=\left(s_{i} t_{j}-t_{i} s_{j}\right) \bmod (\tilde{n}) .
$$

In the remainder of this section we will calculate the commutation relations satisfied by the $Z_{i}$ 's and the constant curvature connection (8). Using their explicit form (20) we find, after some matrix algebra,

$$
Z_{i} Z_{j}=e^{2 \pi i \Theta_{i j}^{\prime}} Z_{j} Z_{i}
$$

where

$$
\Theta^{\prime}=\tilde{n}^{-2} N^{T} Q^{T} \Theta Q N-\tilde{n}^{-1} L
$$

From (23) we see that the algebra generated by the $Z_{i}$ 's is the algebra of functions on the quantum torus with deformation parameters given by $\Theta^{\prime}$. After some further matrix algebra and using the following identities,

$$
\begin{aligned}
Q & =1+2 \pi F \Theta \\
Q^{2} & =1+2 \pi \mathcal{F}_{(0)} \Theta=(1-\widetilde{M} \Theta / \tilde{n})^{-1}, \\
Q^{T} \Theta & =\Theta Q
\end{aligned}
$$

we can rewrite $\Theta^{\prime}$ as a fractional transformation

$$
\Theta^{\prime}=\Lambda(\Theta) \stackrel{\text { def }}{=}(\mathcal{A} \Theta+\mathcal{B})(\mathcal{C} \Theta+\mathcal{D})^{-1}
$$


Here

$$
\Lambda=\left(\begin{array}{ll}
\mathcal{A} & \mathcal{B} \\
\mathcal{C} & \mathcal{D}
\end{array}\right),
$$

and the $d$-dimensional block matrices are given by

$$
\mathcal{A}=\tilde{n}^{-1}\left(N^{T}+L N^{-1} \widetilde{M}\right), \mathcal{B}=-L N^{-1}, \mathcal{C}=-N^{-1} \widetilde{M}, \mathcal{D}=\tilde{n} N^{-1} .
$$

One can check that

$$
\mathcal{A}^{T} \mathcal{D}+\mathcal{C}^{T} \mathcal{B}=1, \mathcal{A}^{T} \mathcal{C}+\mathcal{C}^{T} \mathcal{A}=0, \mathcal{B}^{T} \mathcal{D}+\mathcal{D}^{T} \mathcal{B}=0
$$

and thus $\Lambda$ is an element of $O(d, d \mid \mathbf{R})$, i.e. it satisfies

$$
\Lambda^{T} J \Lambda=J
$$

where

$$
J=\left(\begin{array}{cc}
0 & I_{d} \\
I_{d} & 0
\end{array}\right) .
$$

In the two and three dimensional examples that we will study later, $\Lambda$ is in fact an element of $S O(d, d \mid \mathbf{Z})$. This is the subgroup with determinant one and integer valued entries in the basis where the metric is given by (28). The Weyl spinor representations of $S O(d, d \mid \mathbf{Z})$ are also integral, that is the representation matrices have integer entries. We prove this statement, which is implicit in papers discussing T-duality of Type II string theory, in the appendix. Since the spinor representation of $S O(d, d \mid \mathbf{Z})$ will be used extensively in the following sections we recall that the vector and spinor representations are related by

$$
\mathcal{S}^{-1} \gamma_{s} \mathcal{S}=\Lambda_{s}^{p} \gamma_{p}
$$

and the gamma matrices satisfy

$$
\left\{\gamma_{s}, \gamma_{p}\right\}=2 J_{s p}
$$

Finally, one can show by direct calculation that the commutation relations of the constant curvature connection and $Z_{i}$ have the form

$$
\left[\nabla^{i}, Z_{j}\right]=i H_{j}^{i} Z_{j}
$$


where there is no sum over $j$ and $H=(\tilde{n}-\widetilde{M} \Theta)^{-1} N$. Note that $H$ can also be written in terms of $\Theta$ and some of the block components of $\Lambda$

$$
H^{-1}=\mathcal{C} \Theta+\mathcal{D}
$$

Finally, we present some identities, which will be useful in later sections

$$
\begin{gathered}
H=\tilde{n}^{-1} Q^{2} N, \quad \operatorname{det}(H)=\tilde{n}^{-2} \operatorname{det}\left(Q^{2}\right), \\
\operatorname{det}\left(Q^{2}\right)=\left(1-\frac{\operatorname{tr}(\widetilde{M} \Theta)}{2 \tilde{n}}\right)^{-2} .
\end{gathered}
$$

Note that all the previous relations are valid for tori of arbitrary dimension provided we work on the bundles discussed in Section 3 .

\section{Two Dimensional Solution}

Although the twisted two dimensional case has been discussed extensively in the literature [13, 21, 22, 26], we review it here in a form that readily admits generalization to higher dimensional compactifications.

In the two dimensional case the antisymmetric matrices $\Theta$ and $M$ have the form

$$
\Theta=\left(\begin{array}{cc}
0 & \theta \\
-\theta & 0
\end{array}\right), M=\left(\begin{array}{cc}
0 & m \\
-m & 0
\end{array}\right)
$$

where $\theta$ is the deformation parameter and $m$ is the magnetic flux, which is interpreted as the number of D2 branes wrapping the two-torus.

One can verify that the integers

$$
\left(a^{i}\right)=(\tilde{m}, 0), \quad\left(b^{i}\right)=(0,1),
$$

where $n=q \tilde{n}$ and $m=q \tilde{m}$, satisfy (14). Then choosing $s_{i}=(0,1)$ and $t_{i}=(b, 0)$, where $b$ is an integer such that $a \tilde{n}-b \tilde{m}=1$, we have $N=I_{2}$. One can now use (22) and (26) to find

$$
\Lambda=\left(\begin{array}{cc}
a I_{2} & b \varepsilon \\
-\tilde{m} \varepsilon & \tilde{n} I_{2}
\end{array}\right)
$$


where $\varepsilon$ is a two dimensional matrix with the only nonvanishing entries given by $\varepsilon_{12}=-\varepsilon_{21}=1$. Group elements of the form above are in an $S L(2, \mathbf{Z})$ subgroup of $S O(2,2 \mid \mathbf{Z})$. This subgroup is isomorphic with one of the Weyl spinor representations of $S O(2,2 \mid \mathbf{Z})$. This feature is not generic for higher dimensional compactifications and reflects the fact that $S O(2,2 \mid \mathbf{Z}) \sim$ $S L(2, \mathbf{Z}) \times S L(2, \mathbf{Z})$, so that it is not simple.

The algebra of the $Z_{i}$ 's is then determined by $\Theta^{\prime}$ which is given by the fractional transformation (25). In two dimensions, the $S O(2,2 \mid \mathbf{Z})$ fractional transformation (25) can also be written in the more familiar form, used in [13, 22], as a $S L(2, \mathbf{Z})$ fractional transformation acting on $\theta$

$$
\theta^{\prime}=\frac{a \theta+b}{\tilde{m} \theta+\tilde{n}} .
$$

One can also check that the other $S L(2, \mathbf{Z})$ subgroup, made of elements of the form

$$
\left(\begin{array}{cc}
R & 0 \\
0 & \left(R^{T}\right)^{-1}
\end{array}\right),
$$

acts trivially on $\theta$. This subgroup is generalized to $S L(d, \mathbf{Z})$ in compactifications on a $d$-dimensional torus, and will play in important role later, but only for the two dimensional compactification it leaves $\Theta$ invariant. The $Z_{i}$ 's then obey the following algebra

$$
Z_{1} Z_{2}=e^{2 \pi i \theta^{\prime}} Z_{2} Z_{1} .
$$

As we will see shortly, the rank of the gauge group and the magnetic flux transform in an integral Weyl spinor representation of $S O(2,2 \mid \mathbf{Z})$. Using the creation and annihilation operators introduced in the appendix we can write such a spinor as

$$
n|0\rangle+m a_{1}^{\dagger} a_{2}^{\dagger}|0\rangle .
$$

Using (29) one can show that the spinor representation of (34) transforms 
the above state into $q|0\rangle$. In the Weyl basis we can write the action as

$$
\left(\begin{array}{l}
q \\
0
\end{array}\right)=S\left(\begin{array}{l}
n \\
m
\end{array}\right),
$$

where

$$
S=\left(\begin{array}{cc}
a & -b \\
-\tilde{m} & \tilde{n}
\end{array}\right) .
$$

In Section 8 we will show, employing the expansion of the adjoint section in terms of the $Z_{i}$ generators (16), how to rewrite the original $U(n)$ NCSYM action on a twisted bundle as a $U(q)$ NCSYM action on a trivial quantum bundle over a torus with deformation parameter $\Theta^{\prime}$. The $S L(2, \mathbf{Z})$ transformation, which relates the deformation parameters and the spinors (36) of these two NCSYM, can then be interpreted as a duality transformation inherited from T-duality of Type II string theory. This can be seen as follows. The rank and the bundle of the NCSYM theory determine the D brane charges in string theory. These charges transform in a chiral spinor representation of the target space duality group [39]. Given $n$ and $m$ with greatest common divisor $q$, one can perform a T-duality transformation which takes the original D brane configuration into $q$ D0 branes.

Of course the metric and antisymmetric tensor also transform under this duality, and in the proper limit, which we will explain in detail later, the antisymmetric tensor $B$ transforms separately by fractional transformation just as in (25). Since the parameters $\Theta_{i j}$ of the NCSYM theory are identified with $B_{i j}$, the background expectation value of the NS antisymmetric tensor of the compactified auxiliary string theory, the expected transformation under target space dualities is (25). 


\section{Three Dimensional Solution}

The three dimensional case will be solved by first performing an $S L(3, \mathbf{Z})$ transformation $R$ to bring $M$ in canonical form 9

$$
M=R M^{0} R^{T},
$$

where

$$
M^{0}=\left(\begin{array}{ccc}
0 & 0 & 0 \\
0 & 0 & m \\
0 & -m & 0
\end{array}\right) .
$$

While it is always possible to find such a transformation, (37) does not define it uniquely. We will first find the solution corresponding to $M^{0}$, and then obtain the general solution by using such an $R$.

First note that $M^{0}$ corresponds to a background magnetic field with flux only through the (23) plane, which suggests that the solution should closely resemble the two dimensional one. As before,

$$
\left(a_{0}^{i}\right)=(0, \tilde{m}, 0), \quad\left(b_{0}^{i}\right)=(0,0,1)
$$

satisfy (14). Similarly if we set

$$
\left(s_{i}^{0}\right)=(0,0,1),\left(t_{i}^{0}\right)=(0, b, 0),
$$

we can satisfy (21) with the $N^{0}$ matrix given by

$$
N^{0}=\left(\begin{array}{ccc}
\tilde{n} & 0 & 0 \\
0 & 1 & 0 \\
0 & 0 & 1
\end{array}\right) .
$$

The diagonal entries of $N^{0}$ divided by $\tilde{n}$ have the interpretation of wave numbers. Thus we see that twisting the boundary conditions allows for

\footnotetext{
${ }^{c}$ It is always possible to bring an antisymmetric matrix in canonical form using $S L(3, \mathbf{R})$ but here we need to do this using an integral matrix.
} 
fractional wave numbers in the second and third directions. Using (39) we find

$$
L^{0}=\left(\begin{array}{ccc}
0 & 0 & 0 \\
0 & 0 & -b \\
0 & b & 0
\end{array}\right) .
$$

One can now use (26) to find the $S O(3,3 \mid \mathbf{Z})$ matrix

$$
\Lambda^{0}=\left(\begin{array}{cccccc}
1 & 0 & 0 & 0 & 0 & 0 \\
0 & a & 0 & 0 & 0 & b \\
0 & 0 & a & 0 & -b & 0 \\
0 & 0 & 0 & 1 & 0 & 0 \\
0 & 0 & -\tilde{m} & 0 & \tilde{n} & 0 \\
0 & \tilde{m} & 0 & 0 & 0 & \tilde{n}
\end{array}\right) .
$$

Everything so far is just as in the two dimensional case. Note however that in general $\Theta$ will not be in canonical form, that is, it will not have a form similar to (38).

We can now write the general solution for an arbitrary $M$ as

$$
\begin{gathered}
a^{i}=R_{j}^{i} a_{0}^{j}, \quad b^{i}=R_{j}^{i} b_{0}^{j}, \\
s_{i}=s_{j}^{0}, \quad t_{i}=t_{j}^{0}, \\
N=R N^{0}, \\
\Lambda=\Lambda^{0}\left(\begin{array}{cc}
R^{T} & 0 \\
0 & R^{-1}
\end{array}\right) .
\end{gathered}
$$

Just as in the two dimensional case we can find, using (29), the Weyl spinor representation matrices corresponding to (40) and (41)

$$
S^{0}=\left(\begin{array}{cccc}
a & -b & 0 & 0 \\
-\tilde{m} & \tilde{n} & 0 & 0 \\
0 & 0 & 1 & 0 \\
0 & 0 & 0 & 1
\end{array}\right),
$$




$$
S=S^{0}\left(\begin{array}{cc}
1 & 0 \\
0 & R^{T}
\end{array}\right) .
$$

The rank of the group and the magnetic flux matrix $M$ define a state in the Weyl spinor Fock space

$$
n|0\rangle+\frac{1}{2} M^{i j} a_{i}^{\dagger} a_{j}^{\dagger}|0\rangle
$$

Now one can check that $S$ acts on this spinor as

$$
\left(\begin{array}{l}
q \\
0 \\
0 \\
0
\end{array}\right)=S\left(\begin{array}{c}
n \\
M^{23} \\
M^{31} \\
M^{12}
\end{array}\right) .
$$

As we will see later this can be used to relate the original theory to a $U(q)$ theory on a trivial bundle. In the appendix we show that the Weyl spinor representation of $S O(3,3 \mid \mathbf{Z})$ is in fact isomorphic to $S L(4, \mathbf{Z})$. In this case, in the auxiliary Type IIA string theory, the D0 and D2 branes form $q$ bound states, and the transformation above corresponds to a T-duality transformation that maps the original D brane configuration into a $q$ D0 branes.

\section{Noncommutative Super Yang-Mills Action}

We are now almost ready to write the noncommutative Super Yang-Mills action, but first we need to understand how to perform integration on a noncommutative torus. In the classical case the integral is a linear map that associates to a function its zero mode Fourier coefficient. Similarly for an element of $\mathcal{A}_{\Theta}$ of the form $a=\sum a_{i_{1} i_{2} \ldots i_{d}} U_{1}^{i_{1}} U_{2}^{i_{2}} \ldots U_{d}^{i_{d}}$ we define the integral as

$$
\int d^{d} \sigma a \stackrel{\text { def }}{=}(2 \pi)^{d} a_{00 \ldots 0} .
$$

One can check that this definition has all the desirable properties of the classical integral, such as linearity and translation invariance in $\sigma_{i}$. For defi- 
niteness, in the remainder of this section we will discuss the three dimensional case.

When twisted $U(n)$ theories are considered, it was found in [13, 22] that the integral must be normalized in a particular way to find a duality invariant spectrum. The normalization can also be obtained directly as the Jacobian of a change of integration variables. Note that the integrand, which is the trace of an adjoint section, obeys the following periodicity

$$
\operatorname{tr} \Psi\left(\sigma_{i}\right)=\operatorname{tr} \Psi\left(\sigma_{i}+2 \pi\left(Q^{-1}\right)_{i}^{j}\right) .
$$

Since $\operatorname{tr} \Psi\left(\sigma_{i}\right)$ does not have periodicity $2 \pi$ in $\sigma_{i}$ it can not be expanded in terms of the $U_{i}$ variables. One can define new variables $\widehat{\sigma}_{i}=\sigma_{j} Q_{k}^{j} R_{i}^{k}$ and $\widehat{U}_{i}=e^{\widehat{\sigma}_{i}}$, where $R$ is an arbitrary $S L(3, \mathbf{Z})$ transformation. In the following sections we will take $R$ to be the matrix that brings $M$ into canonical form (37). Then

$$
\int d^{3} \sigma \operatorname{tr} \Psi(\sigma)=\int d^{3} \widehat{\sigma}\left|\operatorname{det}\left(Q^{-1}\right)\right| \operatorname{tr} \Psi\left(\widehat{\sigma} Q^{-1}\right),
$$

where $\operatorname{det}\left(Q^{-1}\right)$ is the Jacobian of the coordinate transformation, and the second integral can now be performed as discussed above, since the integrand has an expansion in terms of the $\widehat{U}_{i}$ variables. Using the expansion (16) of $\Psi$ we obtain

$$
\int d^{3} \sigma \operatorname{tr} \Psi(\sigma)=(2 \pi)^{3} \tilde{n}\left|\operatorname{det}\left(Q^{-1}\right)\right| \sum_{a=1}^{q} \Psi_{000}^{a a} .
$$

The Super Yang-Mills action on a noncommutative three-torus is given by

$$
\begin{aligned}
\mathcal{S}^{U(n)}= & \frac{1}{g_{S Y M}^{2}} \int d t \int d^{3} \sigma \sqrt{\operatorname{det}\left(G^{i j}\right)} \operatorname{tr}\left(\frac{1}{2} G_{i j} \mathcal{F}^{0 i} \mathcal{F}^{0 j}-\right. \\
& \frac{1}{4} G_{i j} G_{k l}\left(\mathcal{F}^{i k}-\mathcal{F}_{(0)}^{i k}\right)\left(\mathcal{F}^{j l}-\mathcal{F}_{(0)}^{j l}\right)+ \\
& \frac{1}{2} \sum_{a} \dot{X}^{a} \dot{X}^{a}-\frac{1}{2} \sum_{a} G_{i j}\left[D^{i}, X^{a}\right]\left[D^{j}, X^{a}\right]+
\end{aligned}
$$




$$
\left.\frac{1}{4} \sum_{a, b}\left[X^{a}, X^{b}\right]\left[X^{a}, X^{b}\right]+\text { fermions }\right),
$$

where $\mathcal{F}^{i j}=i\left[D^{i}, D^{j}\right]$ and $\mathcal{F}_{0}^{i j}=i\left[\nabla^{i}, \nabla^{j}\right]$. We have subtracted the constant part of the field strength in the second line of equation (44). This is equivalent to adding a constant to the Lagrangian, or equivalently to the Hamiltonian, and has the effect of setting the vacuum energy to zero.

For the compactification of the auxiliary Type IIA string theory without wrapped D2 branes, the above action can be obtained directly from the Matrix action. One has to show that the trace over infinite dimensional matrices reduces to a finite dimensional trace and an integral. A formal argument for the commutative case was given in [11] and discussed in detail in [40]. The same argument extends to the noncommutative case. A brief argument was given in [13] showing how to extend this construction when there are D2 branes wrapped on the torus in the auxiliary Type IIA string theory, corresponding to magnetic fluxes in the NCSYM gauge theory. Here we will just make the assumption that the NCSYM action is independent of the D2 brane charges and that adding D2 branes only results in changing the quantum adjoint bundle. We will provide evidence for this in the final section of the paper.

\section{$8 S O(3,3 \mid \mathbf{Z})$ Duality of Super Yang-Mills}

In this section we start with the $U(n)$ NCSYM action (44) on a twisted quantum bundle with magnetic fluxes $M$ and deformation parameter $\Theta$, and we show that after a sequence of field redefinitions it can be rewritten as a $U(q)$ NCSYM action on a trivial bundle over a quantum torus with deformation parameter $\Theta^{\prime}$.

Using the matrix $H$ defined in (31) we make the following constant curvature and field redefinitions

$$
\widehat{\nabla}^{i} \stackrel{\text { def }}{=}\left(H^{-1}\right)_{j}^{i} \nabla^{j}, \widehat{A}^{i} \stackrel{\text { def }}{=}\left(H^{-1}\right)_{j}^{i} A^{j},
$$




$$
\begin{gathered}
\widehat{D}^{i} \stackrel{\text { def }}{=}\left(H^{-1}\right)_{j}^{i} D^{j}, \\
\widehat{\mathcal{F}}^{k l}=\left[\widehat{\nabla}^{k}, \widehat{A}^{l}\right]-\left[\widehat{\nabla}^{l}, \widehat{A}^{k}\right]-i\left[\widehat{A}^{k}, \widehat{A}^{l}\right] .
\end{gathered}
$$

In terms of the new variables the commutator of the constant curvature connection and the $Z_{i}$ 's takes the simple form,

$$
\left[\widehat{\nabla}^{i}, Z_{j}\right]=i \delta_{j}^{i} Z_{j}
$$

and the curvature can be expressed as

$$
\mathcal{F}^{i j}=\mathcal{F}_{(0)}^{i j}+H_{k}^{i} H_{l}^{j} \widehat{\mathcal{F}}^{k l}
$$

One can now rewrite the action in terms of the hatted variables and perform the change of coordinates (43)

$$
\begin{aligned}
\mathcal{S}^{U(n)}= & \frac{1}{g_{S Y M}^{\prime 2}} \int d t \int d^{3} \widehat{\sigma} \sqrt{\operatorname{det}\left(G^{\prime i j}\right)} \frac{1}{\tilde{n}} \operatorname{tr}\left(\frac{1}{2} G_{i j}^{\prime} \widehat{\mathcal{F}}^{0 i} \widehat{\mathcal{F}}^{0 j}-\right. \\
& \frac{1}{4} G_{i j}^{\prime} G_{k l}^{\prime} \widehat{\mathcal{F}}^{i k} \widehat{\mathcal{F}}^{j l}+ \\
& \frac{1}{2} \sum_{a} \dot{X}^{a} \dot{X}^{a}-\frac{1}{2} \sum_{a} G_{i j}^{\prime}\left[\widehat{D}^{i}, X^{a}\right]\left[\widehat{D}^{j}, X^{a}\right]+ \\
& \left.\frac{1}{4} \sum_{a, b}\left[X^{a}, X^{b}\right]\left[X^{a}, X^{b}\right]+\text { fermions }\right) .
\end{aligned}
$$

We have introduced a new gauge coupling and metric given by

$$
\begin{gathered}
g_{S Y M}^{\prime 2}=\tilde{n}\left|\operatorname{det}\left(Q^{-1}\right)\right| g_{S Y M}^{2} \\
G^{\prime i j}=\left(H^{-1}\right)_{k}^{i}\left(H^{-1}\right)_{l}^{j} G^{k l}
\end{gathered}
$$

and used (33) to make these substitutions.

Next we introduce primed variables $\sigma_{i}^{\prime}, U_{i}^{\prime}$ and partial derivatives $\partial^{\prime i}$ satisfying

$$
\left[\sigma_{i}^{\prime}, \sigma_{j}^{\prime}\right]=-2 \pi i \Theta_{i j}^{\prime}
$$




$$
\begin{gathered}
{\left[\partial^{\prime i}, \sigma_{j}^{\prime}\right]=\delta_{j}^{i}, \quad\left[\partial^{\prime i}, \partial^{\prime j}\right]=0,} \\
U_{i}^{\prime} \stackrel{\text { def }}{=} e^{i \sigma_{i}^{\prime}}, \\
U_{i}^{\prime} U_{j}^{\prime}=e^{2 \pi i \Theta_{i j}^{\prime}} U_{j}^{\prime} U_{j}^{\prime} .
\end{gathered}
$$

Comparing the algebra satisfied by $Z_{i}$ and $\widehat{\nabla}^{i}$ on one hand and $U_{i}^{\prime}$ and $\partial_{i}^{\prime}$ on the other, we see that all the commutation relations are the same except that the $\widehat{\nabla}^{i}$ s do not commute while the $\partial^{\prime i}$ s do. The dynamical variables of the theory are the $c$-number coefficients appearing in the expansion (16) of the adjoint sections in terms of $Z_{i}$ 's. Since in the action, the constant curvature covariant derivatives only appear in commutators with the $Z_{i}$ 's and not with each other, substituting $U_{i}^{\prime}$ and $\partial^{\prime i}$ for $Z_{i}$ and $\widehat{\nabla}^{i}$ leaves the dynamics invariant. A similar construction was also considered in [24]. The integral and trace of the $U(n)$ theory can be translated to a $U(q)$ integral using the definition of the integral (42)

$$
\int d^{3} \widehat{\sigma} \frac{1}{\tilde{n}} \operatorname{tr} \Psi\left(Z_{i}\right)=\int d^{3} \sigma^{\prime} \operatorname{tr}_{q} \Psi\left(U_{i}^{\prime}\right)=(2 \pi)^{3} \sum_{a=1}^{q} \Psi_{000}^{a a} .
$$

Making these substitutions we obtain the $U(q)$ action

$$
\begin{gathered}
\mathcal{S}^{U(q)}=\frac{1}{g_{S Y M}^{\prime 2}} \int d t \int d^{3} \sigma^{\prime} \sqrt{\operatorname{det}\left(G^{\prime i j}\right)} \operatorname{tr}_{q}\left(\frac{1}{2} G_{i j}^{\prime}{\mathcal{F}^{\prime 0 i}}^{\prime \mathcal{F}^{0 j}-}\right. \\
\frac{1}{4} G_{i j}^{\prime} G_{k l}^{\prime} \mathcal{F}^{\prime i k} \mathcal{F}^{\prime j l}+ \\
\frac{1}{2} \sum_{a} \dot{X}^{a} \dot{X}^{a}-\frac{1}{2} \sum_{a} G_{i j}^{\prime}\left[D^{\prime i}, X^{a}\right]\left[D^{\prime j}, X^{a}\right]+ \\
\left.\frac{1}{4} \sum_{a, b}\left[X^{a}, X^{b}\right]\left[X^{a}, X^{b}\right]+\text { fermions }\right),
\end{gathered}
$$

where

$$
D^{\prime i} \stackrel{\text { def }}{=} \partial^{\prime i}-i A^{\prime i}, \mathcal{F}^{\prime i j}=i\left[D^{\prime i}, D^{\prime j}\right]
$$

are the $U(q)$ connection and curvature. Thus we have shown that the original $U(n)$ theory is equivalent to a $U(q)$ NCSYM theory with gauge coupling given 
by (45) and defined on a trivial adjoint bundle over a noncommutative torus with deformation parameter $\Theta^{\prime}$ and metric given by (46).

In general two NCSYM theories are dual to each other if there exists an element $\Lambda$ of $S O(3,3 \mid \mathbf{Z})$ with Weyl spinor representation matrix $S$, such that their defining parameters are related as follows

$$
\begin{gathered}
\bar{\Theta}=(\mathcal{A} \Theta+\mathcal{B})(\mathcal{C} \Theta+\mathcal{D})^{-1}, \\
\left(\begin{array}{c}
\bar{n} \\
\bar{M}^{23} \\
\bar{M}^{31} \\
\bar{M}^{12}
\end{array}\right)=S\left(\begin{array}{c}
n \\
M^{23} \\
M^{31} \\
M^{12}
\end{array}\right), \\
\bar{G}^{i j}=(\mathcal{C} \Theta+\mathcal{D})_{k}^{i}(\mathcal{C} \Theta+\mathcal{D})^{j}{ }_{l} G^{k l}, \\
\bar{g}_{S Y M}^{2}=\sqrt{|\operatorname{det}(\mathcal{C} \Theta+\mathcal{D})|} g_{S Y M}^{2},
\end{gathered}
$$

where we used (32) in the last two equations. While $\Theta$ in (47) and the rank and magnetic flux numbers in (48) transform separately and the duality group action can be seen explicitly, the transformation of the gauge coupling and the metric also depends on $\Theta$. Note that $\mathcal{C} \Theta+\mathcal{D}$ satisfies a group property. If $\Lambda_{3}=\Lambda_{2} \Lambda_{1}$ and $\Theta^{\prime}=\Lambda_{1}(\Theta)$ then

$$
\mathcal{C}_{3} \Theta+\mathcal{D}_{3}=\left(\mathcal{C}_{2} \Theta^{\prime}+\mathcal{D}_{2}\right)\left(\mathcal{C}_{1} \Theta+\mathcal{D}_{1}\right)
$$

For a nonvanishing $\bar{n}$ we remove the sign ambiguity that exists when we try to associate to a $S O(3,3 \mid \mathbf{Z})$ transformation its spinor representation matrix, by requiring that $\bar{n}$ is positive. Strictly speaking, one should not consider duality transformations for which $\bar{n}$ vanishes since in this case the description in terms of gauge theories becomes singular.

\section{Target Space Duality}

Next we show that the $S O(3,3 \mid \mathbf{Z})$ duality discussed in the previous section is the realization in NCSYM gauge theories of T-duality in the auxiliary Type 
IIA string theory. This relation is described by the following diagram.

\begin{tabular}{|c|c|c|c|c|}
\hline IIA & $\begin{array}{c}n, \quad M^{i j} \\
g_{s} \\
G_{i j} \\
B_{i j}\end{array}$ & $\longleftrightarrow$ & NCSYM & $\begin{array}{c}U(n), M^{i j} \\
g_{S Y M} \\
G^{i j} \\
\Theta_{i j}=B_{i j}\end{array}$ \\
\hline & $i$ & & & $\uparrow$ \\
\hline IIA & $\begin{array}{c}q, \quad M^{\prime i j}=0 \\
g_{s}^{\prime} \\
G_{i j}^{\prime} \\
B_{i j}^{\prime}\end{array}$ & $\longleftrightarrow$ & NCSYM & $\begin{array}{c}U(q), M^{\prime i j}=0 \\
g_{S Y M}^{\prime} \\
G^{\prime i j} \\
\Theta_{i j}^{\prime}=B_{i j}^{\prime}\end{array}$ \\
\hline
\end{tabular}

The right side of the diagram shows the equivalence described in Section (8). The horizontal arrows represent the Connes, Douglas and Schwarz conjecture [13]. The left side of the diagram contains the string coupling, D brane charges, and compactification moduli of the two auxiliary Type IIA string theories corresponding to the NCSYM's on the right. The additional moduli corresponding to Ramond-Ramond backgrounds were set to zero in this paper and will be considered separately in [41]. Note that the NCSYM metric is the inverse of the Type IIA metric as indicated by the index position, the deformation parameter equals the NS antisymmetric tensor, and the rank and magnetic flux numbers translate into D0 brane number and D2 brane winding. Finally the SYM and string coupling are related by (身).

In the remainder of this section we will calculate the relation between the parameters of the two auxiliary Type IIA string theories. First we describe how the metric, antisymmetric tensor and the string coupling transform under an arbitrary T-duality transformation, and then we take the limit

$$
\alpha^{\prime} \rightarrow 0, \quad G_{i j} \rightarrow 0,
$$


keeping $\alpha^{\prime-2} G_{i j}$ constant. This is the limit proposed by Seiberg and Sen [6, 5] and briefly discussed in the introduction. However, in this limit the auxiliary Type IIA string metric vanishes. Instead we will calculate directly the inverse metric of the NCSYM theory which, after including factors of $\alpha^{\prime}$, is given by $\alpha^{\prime-2} G_{i j}$.

Under the T-duality group $S O(d, d \mid \mathbf{Z})$ the metric and NS antisymmetric tensor transform together by fractional transformations [42]

$$
G^{\prime}+B^{\prime}=(\mathcal{A}(G+B)+\mathcal{B})(\mathcal{C}(G+B)+\mathcal{D})^{-1} .
$$

Using the identification between $\Theta$ and $B$ we have $H^{-1}=\mathcal{C} B+\mathcal{D}$. Then, after some matrix algebra, we can write the symmetric and antisymmetric part of (53) as

$$
\begin{aligned}
G^{\prime} & =H^{T} G\left(1-(H \mathcal{C} G)^{2}\right)^{-1} H, \\
B^{\prime} & =(\mathcal{A} B+\mathcal{B})(\mathcal{C} B+\mathcal{D})^{-1}-H^{T} G H \mathcal{C} G\left(1-(H \mathcal{C} G)^{2}\right)^{-1} H .
\end{aligned}
$$

To derive this we used the fact that $H \mathcal{C}$ is antisymmetric. This can be shown using

$$
(\mathcal{C} B+\mathcal{D})^{-1}=\left(\mathcal{A}-(\mathcal{A} B+\mathcal{B})(\mathcal{C} B+\mathcal{D})^{-1} \mathcal{C}\right)^{T},
$$

which follows from (27). Note that (54) and (55) have simple expansions in $G$. For an elementary T-duality in the $x^{1}$ direction the string coupling constant transforms as

$$
g_{S}^{\prime}=g_{S} G_{11}^{-1 / 2} .
$$

Taking the limit (52) in (55) we can see that the antisymmetric tensor itself transforms by fractional transformation]

$$
B^{\prime}=(\mathcal{A} B+\mathcal{B})(\mathcal{C} B+\mathcal{D})^{-1}
$$

\footnotetext{
${ }^{d}$ We hope there is no confusion between $B$, denoting the NS tensor, and $\mathcal{B}$ which is the upper right block of $\Lambda$.

${ }^{e}$ This is consistent with the fact that the action by fractional transformations preserves the antisymmetry of the matrices.
} 
To find the duality transformation of the metric, we reinstate factors of $\alpha^{\prime}$ in (54) since the $S O(3,3 \mid \mathbf{Z})$ transformations are defined to act on dimensionless fields. Now we can take the limit (52) and to first order in the dimensionless metric $\alpha^{\prime-1} G_{i j}$ we have

$$
\alpha^{\prime-2} G^{\prime}=(\mathcal{C} B+\mathcal{D})^{-T}\left(\alpha^{\prime-2} G\right)(\mathcal{C} B+\mathcal{D})^{-1}
$$

If we make the identification $B=\Theta$, we recognize above the $H$ matrix defined in (32).

Finally using (56), we can also calculate how the string coupling transforms under duality. It was shown in 20] that the $S O(d, d \mid \mathbf{Z})$ group is generated by a set of simple elements. These are written explicitly in the appendix. For each of these generators one can check using (56) that the string coupling transforms as

$$
g_{S}^{\prime}=g_{S}|\operatorname{det}(\mathcal{C} B+\mathcal{D})|^{-1 / 2}
$$

In fact (59) is true for an arbitrary transformation because $\mathcal{C} B+\mathcal{D}$ satisfies the group property (51).

Comparing the T-duality relations (57), (58) and (59) with the NCSYM duality relations (47), (49) and (50), using (4) to relate the string and gauge couplings, we see that indeed the two dualities coincide.

\section{Acknowledgments}

This work was supported in part by the Director, Office of Energy Research, Office of High Energy and Nuclear Physics, Division of High Energy Physics

of the U.S. Department of Energy under Contract DE-AC03-76SF00098 and in part by the National Science Foundation under grant PHY-95-14797. 


\section{Appendix}

In the first part of this appendix we show that the Weyl spinor representations of $S O(d, d \mid \mathbf{Z})$ are integral, i.e. have matrix elements which are integers. In the final part, we show that for $d=3$ the Weyl spinor representation is in fact isomorphic to $S L(4, \mathbf{Z})$.

The gamma matrices obeying (30), where the metric has the form (28), are already, up to normalization, the standard creation and annihilation operators used to generate the Fock space for Dirac spinors in the Weyl basis. These are defined as

$$
a_{i}^{\dagger}=\gamma_{i} / \sqrt{2}, \quad a_{i}=\gamma_{d+i} / \sqrt{2}
$$

and satisfy the canonical anti-commutation relations

$$
\left\{a_{i}, a_{j}^{\dagger}\right\}=\delta_{i j}, \quad\left\{a_{i}, a_{j}\right\}=\left\{a_{i}^{\dagger}, a_{j}^{\dagger}\right\}=0, \quad i, j=1, \ldots, d .
$$

As usual, the Dirac spinor and vector representations are related through formula (29) in the main text

$$
\mathcal{S}^{-1} \gamma_{s} \mathcal{S}=\Lambda_{s}^{p} \gamma_{p}
$$

To prove that the Weyl spinor representations are integral we use a theorem presented in 24] where it was shown that the whole group $S O(d, d \mid \mathbf{Z})$ is generated by a special subset of group elements. We will construct explicitly the Weyl spinor representation matrices corresponding to the group elements in that subset and show that they are integral. The subset contains three types of elements. The first type are generators of the form

$$
\left(\begin{array}{cc}
I_{d} & n \\
0 & I_{d}
\end{array}\right), \quad n^{T}=-n .
$$

The second type of generators forming a $S L(d, \mathbf{Z}) \times \mathbf{Z}_{2}$ subgroup have the form

$$
\left(\begin{array}{cc}
R & 0 \\
0 & R^{T^{-1}}
\end{array}\right), \quad \operatorname{det} R= \pm 1
$$


These are the T-duality generators corresponding to a change of basis of the of the compactification lattice.

The final generator is given by

$$
\left(\begin{array}{llllll}
0 & & & 1 & & \\
& 0 & & & 1 & \\
& & I_{d-2} & & & 0_{d-2} \\
1 & & & 0 & & \\
& 1 & & & 0 & \\
& & 0_{d-2} & & & I_{d-2}
\end{array}\right) .
$$

It corresponds to T-duality along the $x^{1}$ and $x^{2}$ coordinates. The full duality group is in fact $O(d, d \mid \mathbf{Z})$ but here we only consider its restriction to $S O(d, d \mid \mathbf{Z})$ which is the subgroup that does not exchange Type IIA and IIB. The full T-duality group is then obtained by adding to the above list one more generator corresponding to T-duality in a single direction.

Using (29) one can check that the Dirac spinor representation corresponding to the first type of generator (60) is

$$
\exp \left(\frac{1}{2} n_{i j} a_{i} a_{j}\right)
$$

This has a finite expansion and is manifestly integer valued in the standard Fock space basis obtained by acting with the creation operators on a vacuum state.

One can prove that the full $S L(d, \mathbf{Z})$ group is generated by its $S L(2, \mathbf{Z})_{i j}$ subgroups acting on the $x^{i}$ and $x^{j}$ coordinates. We can use this to find the spinor representation matrices corresponding to generators of the second type (61). Since each $S L(2, \mathbf{Z})_{i j}$ is generated by its $T_{i j}$ and $S_{i j}$ transformations, which in the $(i j)$ subspace where $i<j$ have the form

$$
\left(\begin{array}{ll}
1 & 1 \\
0 & 1
\end{array}\right),\left(\begin{array}{cc}
0 & -1 \\
1 & 0
\end{array}\right),
$$


it is enough to find the spinor matrices for these generators. The spinor representation of $T_{i j}$ is given by

$$
\exp \left(a_{i} a_{j}^{\dagger}\right)
$$

The exponential (64) has a finite expansion and its matrix elements are integer valued. Similarly the spinor representation of $S_{i j}$ is given by

$$
\exp \left(\frac{\pi}{2}\left(a_{j}^{\dagger} a_{i}-a_{i}^{\dagger} a_{j}\right)\right)
$$

Let us define $A=a_{j}^{\dagger} a_{i}-a_{i}^{\dagger} a_{j}$ for fixed values of $i$ and $j$. In terms of number operators $N_{i}=a_{i}^{\dagger} a_{i}$ we have $A^{2}=-N_{i}-N_{j}+2 N_{i} N_{j}$. Since $N_{i}$ can be either zero or one, $A^{2}$ is zero or minus one. The Fock space can be split into a direct sum of two subspaces, defined by the eigenvalues of $A^{2}$. On the subspace defined by $A^{2}=0$, we also have $A=0$ and thus the spinor representation (65) reduces to the identity. On the subspace defined by $A^{2}=$ -1 , the exponential can be written as $\cos (\pi / 2)+A \sin (\pi / 2)=A$. On both subspaces, the representation matrix of the transformation is integer valued. A formula for the spinor representation of the $S_{i j}$ generators which is valid on both subspaces is given by, $1+A+A^{2}$. The second type of generator (61) also contains elements with $\operatorname{det} R=-1$. A spinor transformation corresponding to such a generator is given by

$$
1-2 a_{1}^{\dagger} a_{1}
$$

Finally, the generator (62) has the spinor representation

$$
\exp \left(\frac{\pi}{2}\left(a_{1}-a_{1}^{\dagger}\right)\left(a_{2}-a_{2}^{\dagger}\right)\right)
$$

It has a finite expansion given by $\left(a_{1}-a_{1}^{\dagger}\right)\left(a_{2}-a_{2}^{\dagger}\right)$, which can be obtained using $\left(\left(a_{1}-a_{1}^{\dagger}\right)\left(a_{2}-a_{2}^{\dagger}\right)\right)^{2}=-1$, and in this form it is manifestly integral.

Since the Fock space basis we have used splits into two subsets of definite chirality, it follows that the Weyl spinor representations of $S O(d, d \mid \mathbf{Z})$ are also integral. 
In the remainder of the appendix we show that the Weyl spinor representation of $S O(3,3 \mid \mathbf{Z})$ is isomorphic to $S L(4, \mathbf{Z})$. First note that for the Lie algebra corresponding to the continuous Lie groups we have the equivalence so $(3,3 \mid \mathbf{R}) \cong \operatorname{sl}(4, \mathbf{R})$. The spinor representation of the first group is isomorphic to the fundamental of the second. Since in the first part of the appendix we proved that the spinor representations are integral it is reasonable to expect that they form a subgroup of $S L(4, \mathbf{Z})$. In fact we will show that they are isomorphic to the whole $S L(4, \mathbf{Z})$ group.

We represent the Weyl spinor state $n|0\rangle+\frac{1}{2} M^{i j} a_{i}^{\dagger} a_{j}^{\dagger}|0\rangle$ as the column

$$
\left(\begin{array}{c}
n \\
M^{23} \\
M^{31} \\
M^{12}
\end{array}\right)
$$

Using operators of the form (64) and (65) we generate an $S L(3, \mathbf{Z})$ subgroup of the form

$$
\left(\begin{array}{ll}
1 & 0 \\
0 & R
\end{array}\right)
$$

where $R$ is the same matrix appearing in (61). We will now show that the Weyl spinor representation also contains $S L(2, \mathbf{Z})_{1 i}$ subgroups which act on the first and the $i+1$ entries of the column spinor (68). These subgroups together with (69) generate the entire $S L(4, \mathbf{Z})$ group. The T-duality generator (67), denoted below $T_{12}$, has the Weyl spinor representation

$$
T_{12}=\left(\begin{array}{cccc}
0 & 0 & 0 & -1 \\
0 & 0 & -1 & 0 \\
0 & 1 & 0 & 0 \\
1 & 0 & 0 & 0
\end{array}\right) .
$$


Let us also consider a transformation $G$ given by

$$
G=\left(\begin{array}{cccc}
1 & 0 & 0 & 0 \\
0 & 1 & 0 & 0 \\
0 & 0 & a & b \\
0 & 0 & c & d
\end{array}\right), \quad a d-b c=1,
$$

which is an element of an $S L(2, \mathbf{Z})$ subgroup of elements of the form (69). By conjugating $G$ with the $T_{12}$ generator

$$
T_{12}^{-1} G T_{12}=\left(\begin{array}{cccc}
d & c & 0 & 0 \\
b & a & 0 & 0 \\
0 & 0 & 1 & 0 \\
0 & 0 & 0 & 1
\end{array}\right)
$$

we find an $S L(2, \mathbf{Z})_{12}$ transformation acting on the first and second entries. All the other $S L(2, \mathbf{Z})_{1 i}$ subgroups can be obtained by conjugating (70) with elements of the form (69). Thus we have found Weyl spinor representations generating the entire $S L(4, \mathbf{Z})$ group. In fact the representation is isomorphic to $S L(4, \mathbf{Z})$ since all the spinor representation matrices (63), (64), (65), (66) and (67) are integral and have unit determinant.

\section{References}

[1] L. Susskind, Another Conjecture about M(atrix) Theory, hep-th/9704080

[2] M. Claudson, M. Halpern, Supersymmetric Ground State Wave Functions, Nucl. Phys. B250 (1985) 689

[3] R. Flume, On Quantum Mechanics with Extended Supersymmetry and Nonabelian Gauge Constraints, Ann. Phys. 164 (1985) 189

[4] M. Baake, P. Reinicke, V. Rittenberg, Fierz Identities for Real Clifford Algebras and the Number of Supercharges, J. Math. Phys. 26 (1985) 1070 
[5] A. Sen, D0 Branes on $T^{n}$ and Matrix Theory, Adv. Theor. Math. Phys. 2 (1998) 51-59; hep-th/9709220

[6] N. Seiberg, Why is the Matrix Model Correct?, Phys. Rev. Lett. 79 (1997) 3577-3580; hep-th/9710009

[7] A. Sen, An Introduction to Non-perturbative String Theory, hepth/9802051

[8] E. Witten, Bound States of Strings and p-Branes, Nucl. Phys. B460 (1996) 335-350; hep-th/9510135

[9] M. R. Douglas, D. Kabat, P. Pouliot, S. H. Shenker, D-branes and Short Distances in String Theory, Nucl. Phys. B485 (1997) 85-127; hepth/9608024

[10] T. Banks, W. Fishler, S.H. Shenker, L Susskind, M Theory As A Matrix Model: A Conjecture, Phys. Rev. D55 (1997) 5112-5128; hep-th/9610043

[11] W. Taylor IV, D-brane Field Theory on Compact Spaces, Phys. Lett. B394 (1997) 283; hep-th/9611042

[12] O. J. Ganor, S. Ramgoolam, W. Taylor IV, Branes, Fluxes and Duality in M(atrix)-Theory, Nucl. Phys. 492, (1997) 191-204

[13] A. Connes, M. R. Douglas, A. Schwarz, Noncommutative Geometry and Matrix Theory: Compactification on Tori, JHEP02(1998)003; hepth/9711162

[14] M. R. Douglas, C. Hull, D-branes and the Noncommutative Torus, JHEP02 (1998) 008; hep-th/9711165

[15] P.-M. Ho, Y.-Y. Wu, Y.-S. Wu, Towards a Noncommutative Geometric Approach to Matrix Compactification, hep-th/9712201 
[16] P.-M. Ho, Y.-S. Wu, Noncommutative Gauge Theories in Matrix Theory, hep-th/9801147

[17] R. Casalbuoni, Algebraic Treatment of Compactification on Noncommutative Tori, hep-th/9801170

[18] Y.-K. E. Cheung, M. Krogh, Noncommutative Geometry from 0-branes in a Background B-field, hep-th/9803031

[19] T. Kawano, K. Okuyama, Matrix Theory on Noncommutative Torus, hep-th/9803044

[20] M. Rieffel, A. Schwarz, Morita equivalence of multidimensional noncommutative tori, math.QA/98033057

[21] F. Ardalan, H. Arfaei, M. M. Sheikh-Jabbari, Mixed Branes and M(atrix) Theory on Noncommutative Torus, hep-th/9803067

[22] P.-M. Ho, Twisted Bundle on Quantum Torus and BPS States in Matrix Theory, hep-th/9803166

[23] D. Bigatti, Non commutative geometry and super Yang-Mills theory, hep-th/9804120

[24] A. Schwarz, Morita equivalence and duality, hep-th/9805034

[25] G. Landi, F. Lizzi, R. J. Szabo, String Geometry and Noncommutative Torus, hep-th/9806099

[26] B. Morariu, B. Zumino, Super Yang-Mills on the Noncommutative Torus, hep-th/9807198

[27] F. Ardalan, H. Arfaei, M. M. Sheikh-Jabbari, Noncommutative Geometry from Strings and Branes, hep-th/9810072 
[28] M. A. Rieffel, Induced Representations of $C^{*}$-algebras, Advances Math. 13 (1974) 176-257

[29] M. A. Rieffel, C*-Algebras Associeted with Irrational Rotations, Pacific J. Math. 93 (1981) 415-429

[30] M. A. Rieffel, Morita Equivalence for Operator Algebras, Operator Algebras and Applications, (R. V. Kadison, ed.) Proc. Symp. Pure Math. 38, Amer. Math. Soc., Providence, 1982

[31] M. A. Rieffel, The Cancellation Theorem for Protective Modules over Irrational Rotations $\mathbf{C}^{*}$-Algebras, Proc. London Math. Soc. (3), 47 (1983) 285-302

[32] M. A. Rieffel, Projective Modules over Higher-Dimensional Noncommutative Tori, Can. J. Math., XL No. 2 (1988) 257-338

[33] N. Ishibashi, H. Kawai, Y. Kitazawa, A. Tsuchiya, A Large N Reduced Model as Superstring, Nucl. Phys. B498 (1997) 467-491

[34] A. Connes, Noncommutative Geometry, Academic Press, 1994

[35] D. Bigatti, Noncommutative Geometry for Outsiders. Part 1, hepth/9802129

[36] A. Connes, M. Rieffel, Yang-Mills for noncommutative two-Tori, Operator Algebras and Mathematical Physics (Iowa City, Iowa, 1985) 237-266, Contemp. Math. Oper. Algebra. Math. Phys. 62, AMS 1987

[37] G. 't Hooft, Some Twisted Self-Dual Solutions for the Yang-Mills Equations on a Hypertorus, Commun. Math. Phys. 81 (1981) 267-275

[38] G. 't Hooft, A Property of Electric and Magnetic Flux in Nonabelian Gauge Theories, Nucl. Phys. B153 (1979) 141 
[39] E. Witten, String Theory Dynamics in Various Dimensions, Nucl. Phys. B443 (1995) 85-126; hep-th/9503124

[40] W. Taylor IV, Lectures on D-branes, Gauge Theory and M(atrices), hepth/9801182

[41] D. Brace, B. Morariu, More on Dualities of the Matrix Model from T-Duality of the Type II String, in preparation.

[42] A. Giveon, M. Porrati and E. Rabinovici, Target Space Duality in String Theory, Phys. Rept. 244 (1994) 77-202; hep-th/9401139 